\title{
Quadrupole association and dissociation of hydrogen in the early universe
}

\author{
Robert C Forreyt \\ Department of Physics, Pennsylvania State University, Berks Campus, Reading, \\ PA 19610-6009, USA
}

\begin{abstract}
Radiative association and photodissociation rates are calculated for quadrupole transitions of $\mathrm{H}_{2}$. A complete set of bound and unbound states are included in a self-consistent master equation to obtain steady-state concentrations for a dilute system of hydrogen atoms and molecules. Phenomenological rate constants computed from the steady-state concentrations satisfy detailed balance for any combination of matter and radiation temperature. Simple formulas are derived for expressing the steady-state distributions in terms of equilibrium distributions. The rate constant for radiative association is found to be generally small for all temperature combinations. The photodissociation rate constant for quadrupole transitions is found to dominate the rate constants for other $\mathrm{H}_{2}$ photodestruction mechanisms for $T_{R} \leq 3000 \mathrm{~K}$. Implications for the formation and destruction of $\mathrm{H}_{2}$ in the early universe are discussed.
\end{abstract}




\section{Introduction}

It is widely accepted that hydrogen molecules catalyzed the formation of the first stars in the universe [1 [5]. As primordial condensations formed, the gas density increased and subsequent collisions produced internally excited states which quenched via the emission of electric quadrupole radiation. Although inefficient, the removal of the quadrupole radiation from the condensation allowed gravitational collapse to proceed toward the eventual first star. The probability of a quadrupole transition scales as the fifth power of the difference in internal energy levels [6, 7]. Therefore, unbound and quasibound states which provide large energy differences might play a role in the early stages of cooling. Nevertheless, the possibility that hydrogen atoms could form molecules via the emission of quadrupole radiation has so far been neglected. Considering that the main processes leading to the formation of $\mathrm{H}_{2}$ are known to be quite slow, it is worth investigating the quadrupole association mechanism to see whether it makes a significant contribution to the formation rate. Such a study is particularly compelling in light of recent developments [8] concerning the role of quasibound states in the recombination process. It was shown for conditions of local thermodynamic equilibrium (LTE) that long-lived orbiting resonances can enhance the radiative asssociation (RA) rate constant by several orders of magnitude 9 compared to calculations where they are neglected. Hydrogen interactions allow orbiting resonances whose lifetimes are extremely long (some are comparable to the age of the universe [10]). Such resonances can provide feedback which may further enhance the formation rates when the gas is not in LTE as is the case in the early universe after the matter and radiation temperatures have decoupled. Therefore, in the present work, we provide a complete account of all resonant and non-resonant contributions for the RA process

$$
H+H \rightarrow H_{2}+\nu
$$

where $\nu$ represents the photon released in a quadrupole transition.

The inverse process of photodissociation via quadrupole transitions is also considered. Photodissociation of $\mathrm{H}_{2}$ is believed to be important in the early universe. Direct collapse to a supermassive black hole may occur if there are no hydrogen molecules to promote cooling and fragmentation [11. A strong radiation field, such as may occur after ignition of the first stars, destroys the formation of $\mathrm{H}^{-}$and $\mathrm{H}_{2}^{+}$ which are precursors for the formation of $\mathrm{H}_{2}$. Direct and indirect photodissociation via dipole transitions to the Lyman and Werner states deplete $\mathrm{H}_{2}$ at high energies. The critical value of the Lyman and Werner intensity $J_{21}^{\text {crit }}$ needed for supermassive star formation [12,13 is determined by the relative strength of the rate constants for photodestruction of $\mathrm{H}^{-}, \mathrm{H}_{2}^{+}$, and $\mathrm{H}_{2}$. Because quadrupole radiation allows transitions within the same ground electronic state, the mechanism would apply at both low and high radiation temperatures and perhaps contribute to the depletion of $\mathrm{H}_{2}$.

In order to perform the study, we use the self-consistent quantum kinetic theory developed previously [8] which computes the rate constants from the steady-state solution of a Sturmian master equation. The bound and unbound energy eigenstates of the Sturmian representation form a complete basis set for both the dynamics and kinetics. Consequently, all transitions between bound and unbound states, which include long-lived quasibound and discretized non-resonant states, are accounted for. The summed rate coefficients are then used to provide phenomenological rate constants for an idealized system consisting of dilute hydrogen atoms and molecules which undergo negligible inelastic collisions. The RA and photodissociation rate constants computed in this way are guaranteed to satisfy detailed balance for any combination of matter and radiation temperature. Therefore, these rate constants are suitable for use in an expanded network of chemical processes. In the present work, however, we consider only the quadrupole mechanism for association and dissociation of hydrogen and draw conclusions about its importance in the early universe. 


\section{Theory}

We begin by considering the rate equation

$$
\frac{d}{d t}\left[H_{2}\right]=M_{r}[H]^{2}-M_{d}\left[H_{2}\right]
$$

for a fixed number density of hydrogen nuclei

$$
n_{H}=[H]+2\left[H_{2}\right]
$$

where square brackets indicate the concentration of the enclosed species, and $M_{r}$ and $M_{d}$ are the respective rate constants for recombination and dissociation. The solution to the rate equation may be written

$$
\begin{aligned}
& {\left[H_{2}\right]=\frac{\left(\frac{n_{H}}{2}\right)^{2}\left(1-e^{-\lambda t}\right)}{\alpha+\beta+(\alpha-\beta) e^{-\lambda t}}} \\
& \alpha=\frac{\lambda}{8 M_{r}} \\
& \beta=\frac{4 M_{r} n_{H}+M_{d}}{8 M_{r}} \\
& \lambda=\left(8 M_{r} M_{d} n_{H}+M_{d}^{2}\right)^{1 / 2} .
\end{aligned}
$$

The rate constants in equation (21) are calculated using the quantum kinetic theory described previously [8]. This method requires that the single rate equation (2) be consistent with a Sturmian master equation which contains all possible transitions, including those involving the discretized continuum. In general, the state-to-state transition probability is given by

$$
M_{i \rightarrow j}= \begin{cases}A_{i \rightarrow j}+B_{i \rightarrow j} \bar{J}_{i \rightarrow j}+C_{i \rightarrow j} & E_{i}>E_{j} \\ B_{i \rightarrow j} \bar{J}_{i \rightarrow j}+C_{i \rightarrow j} & E_{j}>E_{i}\end{cases}
$$

where $A$ and $B$ are Einstein coefficients, $C$ is a collisional rate coefficent, and $\bar{J}$ is the average radiation field at the frequency corresponding to a molecular transition between states with energies $E_{i}$ and $E_{j}$. The master equation may be divided into two sets of equations corresponding to bound $(b)$ and unbound $(u)$ states

$$
\begin{aligned}
\frac{d}{d t}\left[H_{2}\left(b_{i}\right)\right] & =\sum_{j}\left(M_{u_{j} \rightarrow b_{i}}\left[H_{2}\left(u_{j}\right)\right]-M_{b_{i} \rightarrow u_{j}}\left[H_{2}\left(b_{i}\right)\right]\right) \\
& +\sum_{j \neq i}\left(M_{b_{j} \rightarrow b_{i}}\left[H_{2}\left(b_{j}\right)\right]-M_{b_{i} \rightarrow b_{j}}\left[H_{2}\left(b_{i}\right)\right]\right) \\
\frac{d}{d t}\left[H_{2}\left(u_{i}\right)\right] & =\sum_{j}\left(M_{b_{j} \rightarrow u_{i}}\left[H_{2}\left(b_{j}\right)\right]-M_{u_{i} \rightarrow b_{j}}\left[H_{2}\left(u_{i}\right)\right]\right) \\
& +\sum_{j \neq i}\left(M_{u_{j} \rightarrow u_{i}}\left[H_{2}\left(u_{j}\right)\right]-M_{u_{i} \rightarrow u_{j}}\left[H_{2}\left(u_{i}\right)\right]\right) \\
& +k_{f \rightarrow u_{i}}^{\text {elastic }}[H]^{2}-\tau_{u_{i}}^{-1}\left[H_{2}\left(u_{i}\right)\right]
\end{aligned}
$$

where $\tau_{u_{i}}$ is the lifetime of the unbound state $u_{i}$, and $k_{f \rightarrow u_{i}}^{\text {elastic }}$ is the two-body elastic scattering rate constant for a discretized free $(f)$ state, which is related to $\tau_{u_{i}}^{-1}$ by the equilibrium constant

$$
K_{u_{i}}^{e q}=\frac{g_{u_{i}} \exp \left(-E_{u_{i}} / k_{B} T\right)}{Q_{H}^{2} Q_{T}} .
$$

$Q_{H}=4$ is the atomic partition function $1, g_{u}=\left(2 I_{u}+1\right)\left(2 j_{u}+1\right)$ is the degeneracy of the unbound state $\left(I_{u}=0\right.$ for para- $\mathrm{H}_{2}$ and $I_{u}=1$ for ortho- $\left.\mathrm{H}_{2}\right), Q_{T}$ is the translational partition function for temperature $T$, and $k_{B}$ is Boltzmann's constant.

$\ddagger$ For the temperatures considered in the present work, the $\mathrm{H}$ atom is assumed to be in the ground state, and the atomic partition function is due to electron and nuclear spin degeneracy. 
Quadrupole association and dissociation of hydrogen in the early universe

The steady-state solution to the master equation yields the rate constants

$$
M_{r}=\frac{\sum_{i j} g_{u_{i}} e^{-E_{u_{i}} / k_{B} T}\left(1+\delta_{u_{i}}\right) M_{u_{i} \rightarrow b_{j}}^{\mathrm{OUT}}}{Q_{H}^{2} Q_{T}}
$$

and

$$
M_{d}=\frac{\sum_{i j} g_{b_{i}} e^{-E_{b_{i}} / k_{B} T}\left(1+\delta_{b_{i}}\right) M_{b_{i} \rightarrow u_{j}}^{\text {ouT }}}{Q_{H_{2}}}
$$

where

$$
Q_{H_{2}}=\sum_{i}\left(1+\delta_{b_{i}}\right) g_{b_{i}} \exp \left(-E_{b_{i}} / k_{B} T\right)
$$

is the molecular partition function, $\delta_{b}$ and $\delta_{u}$ are non-LTE concentration defects which may be obtained from the steady-state concentrations

$$
\begin{aligned}
& 1+\delta_{b_{i}}=\frac{\left(\frac{Q_{H_{2}}\left[H_{2}\left(b_{i}\right)\right]}{\left[H_{2}\right]}\right)_{\mathrm{SS}}}{\left(\frac{Q_{H_{2}}\left[H_{2}\left(b_{i}\right)\right]}{\left[H_{2}\right]}\right)_{\mathrm{LTE}}}=\sum_{j, k, l} \tilde{A}_{b_{i} b_{j}}^{-1} M_{b_{j} \rightarrow u_{k}}^{\mathrm{IN}} \tilde{B}_{u_{k} u_{l}}^{-1} \\
& 1+\delta_{u_{i}}=\frac{\left(\frac{\left[H_{2}\left(u_{i}\right)\right]}{[H]^{2}}\right)_{\mathrm{SS}}}{\left(\frac{\left[H_{2}\left(u_{i}\right)\right]}{[H]^{2}}\right)_{\mathrm{LTE}}}=\sum_{j} \tilde{B}_{u_{i} u_{j}}^{-1}
\end{aligned}
$$

and the matrices $\tilde{A}$ and $\tilde{B}$ and the rate coefficients $M_{i \rightarrow j}^{\mathrm{IN}}$ and $M_{i \rightarrow j}^{\text {oup }}$ are defined below. Equations (15) and (16) give the identity

$$
1=\frac{\sum_{i j} g_{u_{i}} e^{-E_{u_{i}} / k_{B} T}\left(1+\delta_{u_{i}}\right) M_{u_{i} \rightarrow b_{j}}^{\text {OUT }}}{\sum_{i j} g_{b_{i}} e^{-E_{b_{i}} / k_{B} T}\left(1+\delta_{b_{i}}\right) M_{b_{i} \rightarrow u_{j}}^{\text {OUT }}}
$$

so that the ratio

$$
\frac{M_{r}}{M_{d}}=\frac{\left[H_{2}\right]}{[H]^{2}}=\frac{Q_{H_{2}}}{Q_{H}^{2} Q_{T}}
$$

reduces to the statistical Saha relation when all $\delta_{b}=\delta_{u}=0$. The matrices $\tilde{A}$ and $\tilde{B}$ which need to be inverted are obtained from equations (9) and (10) and are given by

$$
\begin{aligned}
\tilde{A}_{b_{i} b_{j}} & =\delta_{i j}\left(\sum_{k \neq i} M_{b_{i} \rightarrow b_{k}}^{\mathrm{OUT}}+\sum_{k} M_{b_{i} \rightarrow u_{k}}^{\mathrm{OUT}}\right)-\left(1-\delta_{i j}\right) M_{b_{i} \rightarrow b_{j}}^{\mathrm{IN}} \\
\tilde{B}_{u_{i} u_{j}} & =\delta_{i j}\left\{1+\tau_{u_{i}}\left(\sum_{k \neq i} M_{u_{i} \rightarrow u_{k}}^{\mathrm{OUT}}+\sum_{k} M_{u_{i} \rightarrow b_{k}}^{\mathrm{OUT}}\right)\right\} \\
& -\tau_{u_{i}}\left\{\left(1-\delta_{i j}\right) M_{u_{i} \rightarrow u_{j}}^{\mathrm{IN}}+\sum_{k, l} M_{u_{i} \rightarrow b_{k}}^{\mathrm{IN}} \tilde{A}_{b_{k} b_{l}}^{-1} M_{b_{l} \rightarrow u_{j}}^{\mathrm{IN}}\right\} .
\end{aligned}
$$

In the present work, we exclude inelastic collisions so that $C_{i \rightarrow j} \equiv 0$ in equation (8). Assuming a complete redistribution of frequencies [14, the average radiation field may be written in terms of the Planck black-body radiation function, $P_{i \rightarrow j}$, and a line source function, $S_{i \rightarrow j}$, as follows [15]

$$
\bar{J}_{i \rightarrow j}\left(T_{M}, T_{R}\right)=\left(1-\beta_{i j}\right) S_{i \rightarrow j}\left(T_{M}\right)+\beta_{i j} P_{i \rightarrow j}\left(T_{R}\right)
$$

where $\beta_{i j}$ is the escape probability for a photon associated with the $i \rightarrow j$ transition, and the dependence of the matrix elements on matter temperature $T_{M}$ and radiation temperature $T_{R}$ is now shown. The line source function may be written

$$
S_{i \rightarrow j}\left(T_{M}\right)=F\left(\nu_{i j}\right)\left(\frac{g_{i} n_{j}}{g_{j} n_{i}}-1\right)^{-1}
$$


where $n_{i}$ and $n_{j}$ are level densities, $\nu_{i j}$ is the frequency of the line photon, and $F(\nu)$ is the usual Planck function coefficient. Because the source function (22) is derived for conditions of statistical equilibrium, it does not contribute to the matrix elements in the steady-state equations (19) and (20). These matrices are then obtained from the Planck function and the Maxwell-Boltzmann factor arising from elastic collisions and may be written as

$$
M_{i \rightarrow j}^{\mathrm{IN}}\left(T_{M}, T_{R}\right)=\frac{g_{j}}{g_{i}} e^{-\left(E_{j}-E_{i}\right) / k_{B} T_{M}} M_{j \rightarrow i}^{\mathrm{ouT}}\left(T_{R}\right)
$$

and

with

$$
M_{i \rightarrow j}^{\mathrm{OuT}}\left(T_{R}\right)=\beta_{i j} R_{i \rightarrow j}\left(T_{R}\right)
$$

$$
R_{i \rightarrow j}\left(T_{R}\right)=\frac{A_{i \rightarrow j}}{1-e^{-\left(E_{i}-E_{j}\right) / k_{B} T_{R}}} .
$$

There is no $T_{M}$-dependence in the outgoing rate coefficient (24) due to the assumption that inelastic collisions are negligible. In order to determine $\delta_{b_{i}}$ and $\delta_{u_{i}}$, it is necessary to compute $\tilde{A}$ and $\tilde{B}$. In practical computations, equations (19) and (20) tend to suffer round-off errors, particularly at low temperatures where the probability for upward transitions can be very small. For such conditions, the inversion of $\tilde{A}$ may be handled analytically

$$
\begin{aligned}
y_{b_{i} u_{j}} & =\sum_{k} \tilde{A}_{b_{i} b_{k}}^{-1} M_{b_{k} \rightarrow u_{j}}^{\mathrm{IN}} \\
& \approx \frac{e^{-E_{b_{i}}\left(1 / k_{B} T_{R}-1 / k_{B} T_{M}\right)} \sum_{k} g_{b_{k}} e^{-E_{b_{k}} / k_{B} T_{M}} M_{b_{k} \rightarrow u_{j}}^{\mathrm{IN}}}{\sum_{k l} g_{b_{k}} e^{-E_{b_{k}} / k_{B} T_{R}} M_{b_{k} \rightarrow u_{l}}^{\text {OUT }} \delta_{I_{i} I_{k}}} .
\end{aligned}
$$

The $\delta$-function in the denominator arises from the impossibility of an ortho-para transition for quadrupole radiation. If three-body collisions were included in $M_{i \rightarrow j}$, equation (27) would need to be modified to include inelastic and exchange reactions. This problem was considered previously [8] where it was shown that all of the unbound states reach a steady-state concentration that is well-approximated by a Boltzmann distribution whenever three-body collisions are important. In the present work, the approximate formula (27) is expected to be reliable when $M_{b \rightarrow u}^{\text {OUT }}$ is less than $M_{b \rightarrow b^{\prime}}^{\text {ouT }}$. This condition, which is nearly always met, yields the result

$$
1+\delta_{b_{i}}=C_{I_{i}} e^{-E_{b_{i}}\left(1 / k_{B} T_{R}-1 / k_{B} T_{M}\right)}
$$

where

$$
C_{I_{i}}=\frac{\sum_{j k} g_{u_{j}} e^{-E_{u_{j}} / k_{B} T_{M}}\left(1+\delta_{u_{j}}\right) M_{u_{j} \rightarrow b_{k}}^{\text {OUT }} \delta_{I_{i} I_{j}}}{\sum_{j k} g_{u_{j}} e^{-E_{u_{j}} / k_{B} T_{R}} M_{u_{j} \rightarrow b_{k}}^{\text {OUT }} \delta_{I_{i} I_{j}}}
$$

is a symmetry constant for para- $\mathrm{H}_{2}$ and ortho- $\mathrm{H}_{2}$ which depends only on $T_{M}$ and $T_{R}$. This allows equation (13) to be written

$$
M_{d}=\frac{\sum_{i j} C_{I_{i}} g_{b_{i}} e^{-E_{b_{i}} / k_{B} T_{R}} M_{b_{i} \rightarrow u_{j}}^{\text {OuT }}}{\sum_{i} C_{I_{i}} g_{b_{i}} e^{-E_{b_{i}} / k_{B} T_{R}}} .
$$

All of the $T_{M}$-dependence in the photodissociation rate constant (30) is contained in the symmetry constant. If $C_{I}$ is approximately the same for both nuclear symmetries, then it is a good approximation to use a Boltzmann distribution of states for $T=T_{R}$. The $\delta_{u_{i}}$ may be obtained by substituting equation (27) into equation (20) to yield

$$
\begin{aligned}
\tilde{B}_{u_{i} u_{j}} & =\delta_{i j}\left\{1+\tau_{u_{i}}\left(\sum_{k \neq i} M_{u_{i} \rightarrow u_{k}}^{\text {OUT }}+\left(1-\epsilon_{i}\right) \sum_{k} M_{u_{i} \rightarrow b_{k}}^{\text {OUT }}\right)\right\} \\
& -\left(1-\delta_{i j}\right) \tau_{u_{i}}\left(M_{u_{j} \rightarrow u_{i}}^{\text {OUT }}+\epsilon_{i} \sum_{k} M_{u_{j} \rightarrow b_{k}}^{\text {OUT }}\right) \\
& \times \frac{g_{u_{j}}}{g_{u_{i}}} e^{-\left(E_{u_{j}}-E_{u_{i}}\right) / k_{B} T_{M}}
\end{aligned}
$$


where

$$
\epsilon_{i}=\frac{g_{u_{i}} e^{-E_{u_{i}} / k_{B} T_{R}} \sum_{k} M_{u_{i} \rightarrow b_{k}}^{\text {OUT }}}{\sum_{j} g_{u_{j}} e^{-E_{u_{j}} / k_{B} T_{R}} \sum_{k} M_{u_{j} \rightarrow b_{k}}^{\text {OUT }} \delta_{I_{i} I_{j}}} .
$$

Equations (16), (31), and (32) may be used to express the system of equations for the non-LTE defects in the form

$$
1+\delta_{u_{i}}=\frac{1+\tau_{u_{i}} \Psi_{i}^{\mathrm{IN}}}{1+\tau_{u_{i}} \Psi_{i}^{\mathrm{OUT}}}
$$

where

$$
\begin{aligned}
\Psi_{i}^{\mathrm{IN}} & =\sum_{j \neq i}\left(1+\delta_{u_{j}}\right) \frac{g_{u_{j}}}{g_{u_{i}}} e^{-\left(E_{u_{j}}-E_{u_{i}}\right) / k_{B} T_{M}} \\
& \times\left(M_{u_{j} \rightarrow u_{i}}^{\mathrm{OUT}}+\epsilon_{i} \sum_{k} M_{u_{j} \rightarrow b_{k}}^{\mathrm{OUT}}\right)
\end{aligned}
$$

and

$$
\Psi_{i}^{\mathrm{OUT}}=\sum_{k \neq i} M_{u_{i} \rightarrow u_{k}}^{\mathrm{OUT}}+\left(1-\epsilon_{i}\right) \sum_{k} M_{u_{i} \rightarrow b_{k}}^{\mathrm{OUT}} .
$$

In the limiting case $T_{R} \rightarrow 0$, equation (32) yields $\epsilon_{1}=1$ for the lowest energy unbound state $u_{1}$ and $\epsilon_{i}=0$ for all states with energy $E_{u_{i}}>E_{u_{1}}$. If $\epsilon_{i}$ is set to zero for all $i$, then the above formulas reduce to ones given previously [8]

$$
\Psi_{i}^{\mathrm{IN}}=\sum_{j>i}\left(1+\delta_{u_{j}}\right) \frac{g_{u_{j}}}{g_{u_{i}}} e^{-\left(E_{u_{j}}-E_{u_{i}}\right) / k_{B} T_{M}} A_{u_{j} \rightarrow u_{i}}
$$

and

$$
\Psi_{i}^{\mathrm{OUT}}=\sum_{j<i} A_{u_{i} \rightarrow u_{j}}+\sum_{j} A_{u_{i} \rightarrow b_{j}}
$$

which may be solved iteratively starting with the highest energy quasibound state and assuming that $\delta_{u_{j}}=0$ for all unbound states that are not quasibound. If $\delta_{u_{j}}=-1$ for all unbound states with $j>i$, then the formula

$$
1+\delta_{u_{i}}=\frac{1}{1+\tau_{u_{i}}\left(\sum_{j<i} A_{u_{i} \rightarrow u_{j}}+\sum_{j} A_{u_{i} \rightarrow b_{j}}\right)}
$$

is obtained which may be substituted into equation (12) to yield the commonly-used Bain and Bardsley formula [16] for resonant radiative association.

\section{Results}

The bound and unbound states of $\mathrm{H}_{2}$ were obtained by solving the radial Schrödinger equation

$$
\left[\frac{1}{2 \mu} \frac{d^{2}}{d r^{2}}-\frac{j(j+1)}{2 \mu r^{2}}-v(r)+E_{v j}\right] \chi_{v j}(r)=0,
$$

where $\mu$ is the reduced mass and $v(r)$ is the $\mathrm{H}_{2}$ potential of Schwenke [10. The notation $b$ and $u$ is understood to mean a unit-normalized bound or unbound energy eigenstate characterized by the pair of quantum numbers $(v, j)$ where $v$ and $j$ are the vibrational and rotational quantum numbers for the eigenstate $\chi_{v j}$. These eigenstates were obtained by diagonalization in an orthonormal $L^{2}$ Sturmian basis set consisting of Laguerre polynomial functions as described previously [17]. The vibrational quantum number for an unbound eigenstate corresponds to the quadrature index and only has meaning with respect to the scale factor and number of basis functions. 
The Einstein A-coefficient for a radiative transition between an initial state $i$ and a final state $f$ is given by [6, 7]

$$
A_{i \rightarrow f}=1.4258 \times 10^{4}\left(E_{i}-E_{f}\right)^{5}\left|\left\langle\chi_{i}|Q(R)| \chi_{f}\right\rangle\right|^{2} f\left(j_{i}, j_{f}\right) \mathrm{s}^{-1}
$$

where $R$ is the internuclear distance, $Q(R)$ is the quadrupole moment, $\chi_{i}$ and $\chi_{f}$ are the energy eigenfunctions of the initial and final states, and the branching ratio is given by

$$
f\left(j_{i}, j_{f}\right)= \begin{cases}\frac{3\left(j_{i}+1\right)\left(j_{i}+2\right)}{2\left(2 j_{i}+1\right)\left(2 j_{i}+3\right)} & j_{f}=j_{i}+2 \\ \frac{j_{i}\left(j_{i}+1\right)}{\left(2 j_{i}-1\right)\left(2 j_{i}+3\right)} & j_{f}=j_{i} \\ \frac{3 j_{i}\left(j_{i}-1\right)}{2\left(2 j_{i}-1\right)\left(2 j_{i}+1\right)} & j_{f}=j_{i}-2\end{cases}
$$

The Sturmian representation was tested by computing the A-coefficients for all bound to bound transitions using the quadrupole moment provided by Wolniewicz et al. [7] Excellent agreement was found for all of the tabulated transitions.

Equation (40) also applies to transitions involving resonant and non-resonant unbound states. Tables 1-4 provide an extension of the published tables [7] to include transitions between bound and quasibound states. Only narrow quasibound states that are well-represented by a single Sturmian eigenstate are included in the tables. Figure 1 shows the cumulative radiative width

$$
\Gamma_{u}=\sum_{b} A_{u \rightarrow b}
$$

for para- $\mathrm{H}_{2}$ and ortho- $\mathrm{H}_{2}$ formation. The sharp resonances due to long-lived quasibound states are clearly evident. In virtually all previous work on RA, extremely narrow resonances of this kind have been neglected, either because they are difficult to resolve using a grid-based method or because of an assumed breakdown of perturbation theory [18 20]. Recently, it was shown [9] that such resonances increased the LTE rate constant for $\mathrm{SiO}$ formation by $\sim 100$ times compared to calculations where the resonances were neglected. A similar observation [8] was made for the RA rate constant for $\mathrm{CH}^{+}$where it was also predicted that the non-LTE defects could contribute to a further increase (or decrease) compared to the LTE values.

To explore this possibility for $\mathrm{H}_{2}$, we computed $M_{i \rightarrow j}^{\mathrm{IN}}$ and $M_{i \rightarrow j}^{\text {OUT }}$ in order to evaluate the non-LTE defects. We assume $\beta_{i j}=1$ which means there is no distortion of the background blackbody radiation field. This assumption is convenient but not necessary - the formulation works as written for an optically thick gas so long as the complete redistribution of frequencies [14] is assumed and the escape probabilities are calculated using the line source function (22). Figures 2-5 show the non-LTE defects for the four longest-lived quasibound states of hydrogen. The tunneling widths 10 for these states are $1.1 \times 10^{-21}, 8.2 \times 10^{-25}, 1.5 \times 10^{-18}$, and $6.0 \times 10^{-34} \mathrm{~cm}^{-1}$ for $j=24,29,31$, and 32 , respectively. The curves were computed using the approximate formula (31) and compared with the exact equation (20) for a range of temperatures where the inversion of equation (19) was numerically stable. Excellent agreement was found in all of the comparisons. As expected, the plots show $\delta_{u}=0$ in the LTE limit $T_{R}=T_{M}$. Complete removal of long-lived quasibound states corresponds to $\delta_{u}=-1$. The top panels of Figures 2-5 show that this condition is approached for low values of $T_{R}$ when $T_{M}>T_{R}$. The depletion is greatest for the quasibound state with the smallest tunneling width $(j=32)$. For the largest tunneling width shown $(j=31)$, the depletion is not as complete - the steady-state population is about $8 \%$ of its LTE value (see Figure 4). For the majority of quasibound states that are not shown, the steady-state and LTE populations are nearly identical $\left(\delta_{u} \approx 0\right)$ for all temperatures.

The middle and lower panels of Figures 2-5 show that $\delta_{u}>0$ for intermediate and large values of $T_{R}$ when $T_{M}>>T_{R}$. For para- $\mathrm{H}_{2}$, the curves increase with $T_{R}$ 
before reaching their largest values at $T_{R} \approx 200-300 \mathrm{~K}$ where they begin to decrease. For ortho- $\mathrm{H}_{2}$, the pattern is similar, however, the curves reach their largest values at $T_{R} \approx 500 \mathrm{~K}$. The lower panels for both para- $\mathrm{H}_{2}$ and ortho- $\mathrm{H}_{2}$ show the curves uniformly decreasing for $T_{R} \geq 1000 \mathrm{~K}$. Interestingly, Figures $2-5$ show that $\delta_{u}>>0$ when $T_{M}<<T_{R}$ which shows that the steady-state density of quasibound states is much greater than the LTE density. This suggests that there may be a significant enhancement in the formation rate constant when $T_{M}<T_{R}$.

In order to see the effect of the steady-state populations, we plot the LTE and non-LTE rate constants in Figure 6. The top panel shows the para- $\mathrm{H}_{2}$ and ortho- $\mathrm{H}_{2}$ contributions to the LTE rate constant. The large hump centered around $1 \mathrm{~K}$ is due to a low energy $j=4$ resonance which has a tunneling lifetime of $8.4 \times 10^{-6} \mathrm{~cm}^{-1}[10$. Ortho- $\mathrm{H}_{2}$ formation begins to dominate at temperatures above $100 \mathrm{~K}$. The total LTE rate constant shows a broad maximum of $\sim 10^{-28} \mathrm{~cm}^{3} \mathrm{~s}^{-1}$ near $500 \mathrm{~K}$. The middle and lower panels of Figure 6 show how the non-LTE populations affect the rate constant for various $T_{R}$. For para- $\mathrm{H}_{2}$, the steady-state and LTE contributions are the same for $T_{M}<50 \mathrm{~K}$ when $T_{R}=10 \mathrm{~K}$. As $T_{M}$ is increased beyond $50 \mathrm{~K}$, the curve for $T_{R}=10 \mathrm{~K}$ drops below the LTE curve. Results for lower values of $T_{R}$ were found to be essentially the same as the $T_{R}=10 \mathrm{~K}$ curve and are not shown. For ortho- $\mathrm{H}_{2}$, the steady-state and LTE contributions are very nearly the same when $T_{M}$ and $T_{R}$ are both less than $100 \mathrm{~K}$. This is due to the somewhat larger energies for the ortho- $\mathrm{H}_{2}$ quasibound states compared to the $j=4$ resonance. As $T_{R}$ increases, the rate constants for both nuclear symmetries increase due to stimulated emission. The non-LTE rate constants may be larger or smaller than their LTE values due the behavior of $\delta_{u}$ shown in Figures 2-5.

The full set of $\delta_{u}$ may be used to compute the symmetry constants $C_{I}$ defined by equation (29). These constants allow convenient computation of the non-LTE concentration defects $\delta_{b}$ from equation (28) and the photodissociation rate constant from equation (30). Numerical results confirmed that $C_{I}$ is indeed a constant for all combinations of vibrational and rotational states of a given symmetry. The top panel of Figure 7 shows $C_{I}$ as a function of $T_{M}$ for $T_{R}=1000,2000,5000$, and $10000 \mathrm{~K}$ using solid lines for $I=0$ and dashed lines for $I=1$. A significant $T_{M}$-dependence may be seen for these constants, however, there is only a weak dependence on the symmetry $I$, particularly at high temperatures. Therefore, the $C_{I}$ in equation (30) approximately drops out which eliminates the $T_{M}$-dependence of the dissociation rate constant $M_{d}$. This weak $T_{M}$-dependence is again due to the assumption that inelastic collisions are negligible which causes the general rate constant $M_{d}$ defined by equation (13) to be equal to the photodissociation rate constant (30). In this limit, the steady-state distribution of bound states at temperature $T_{M}$ is well-approximated by a Boltzmann equilibrium distribution at temperature $T_{R}$.

While a Boltzmann distribution of molecular states at temperature $T_{R}$ is a good approximation when inelastic collisions are negligible, it should be noted that there is a scale change associated with the partition functions

$$
\Lambda\left(T_{R}, T_{M}\right) \equiv \frac{Q_{H_{2}}^{S S}}{Q_{H_{2}}^{L T E}}
$$

which yields

$$
\left(\frac{M_{r}}{M_{d}}\right)_{S S}=\Lambda\left(T_{R}, T_{M}\right)\left(\frac{M_{r}}{M_{d}}\right)_{L T E} .
$$

The bottom panel of Figure 7 shows the scale factor for the quadrupole mechanism as a function of temperature. As expected, the figure shows $\Lambda=1$ when $T_{R}=T_{M}$. However, there is a large variation in $\Lambda$ when the temperatures are not the same. When $T_{R}<T_{M}$, the scale factor is larger than unity, and equation (44) shows that the steady-state ratio $M_{r} / M_{d}$ is larger than the LTE ratio. This change of scale is important whenever radiative transitions are more probable than inelastic collisions. In such cases, the usual detailed balance assumption $\Lambda=1$ cannot be used. 
Figure 8 shows the steady-state rate constants for quadrupole association and dissociation of hydrogen. The $M_{r}$ curves displayed in the top panel are reminiscent of the RA rate constants for $\mathrm{H}$ and D calculated by Stancil and Dalgarno [21] which included stimulated plus spontaneous radiative dipole transitions. In the present case, the rate constants for $\mathrm{H}_{2}$ formation are about 100 times smaller than for $\mathrm{HD}$ formation. The modification in the rate constants due to the non-LTE concentration defects $\delta_{u}$ is seen to be significant, however, the net effect is still very small due to the weak radiative coupling. The bottom panel of Figure 8 shows the dissociation rate constant $M_{d}$ as a function of $T_{R}$. There is no dependence on $T_{M}$ at the level of resolution of the plot. Also shown are rate constants for photoionization

$$
\mathrm{H}_{2}+\nu \rightarrow \mathrm{H}_{2}^{+}+e^{-}
$$

and indirect photodissociation

$$
\mathrm{H}_{2}+\nu \rightarrow \mathrm{H}+\mathrm{H}
$$

which occurs via dipole transitions to the Lyman and Werner systems. Both of these rate constants are taken from the fitting formulas given in Coppola et al. [22]. The photodissociation rate constant for quadrupole radiation dominates the other $\mathrm{H}_{2}$ photodestruction rate constants for $T_{R} \leq 3000 \mathrm{~K}$. For a more general radiation field comprised of low energy photons, the quadrupole transitions would likewise provide the strongest mechanism for photodestruction of $\mathrm{H}_{2}$. Because the first luminous sources produced diluted blackbody fields with $T_{R}>10,000 \mathrm{~K}$, the quadrupole mechanism is not expected to make a significant contribution to the suppression of $\mathrm{H}_{2}$ molecules.

\section{Discussion}

With the rate constants described above, we may assess the significance of quadrupole association and dissociation on the formation of $\mathrm{H}_{2}$ in the early universe. The rate constants shown in Figure 8 are extremely small and would be unimportant in all but the most extreme environments. The $\mathrm{H}^{-}$sequence

$$
\begin{aligned}
& H+e^{-} \rightarrow H^{-}+\nu \\
& H^{-}+H \rightarrow H_{2}+e^{-}
\end{aligned}
$$

is known to be the dominant $\mathrm{H}_{2}$ formation mechanism in the early universe when the radiation field was weak [4,5]. For redshift $z>100$, the cosmic background radiation destroys $\mathrm{H}^{-}$through the reverse of process (47). In this case, the RA processes

$$
\begin{aligned}
& H^{+}+H \rightarrow H_{2}^{+}+\nu \\
& H^{+}+H e \rightarrow \mathrm{HeH}^{+}+\nu
\end{aligned}
$$

followed by

$$
\begin{aligned}
& \mathrm{HeH}^{+}+\mathrm{H} \rightarrow \mathrm{H}_{2}^{+}+\mathrm{He} \\
& \mathrm{H}_{2}^{+}+\mathrm{H} \rightarrow \mathrm{H}_{2}+\mathrm{H}^{+}
\end{aligned}
$$

are the dominant $\mathrm{H}_{2}$ formation mechanisms. These mechanisms are also destroyed by the cosmic background radiation at high redshifts through the reverse of processes (49) and (50). Because the binding energies of $\mathrm{H}_{2}^{+}$and $\mathrm{HeH}^{+}$are smaller than the binding energy of $\mathrm{H}_{2}$, they are more easily photodissociated than $\mathrm{H}_{2}$, and the quadrupole mechanisms may be significant. To see whether this is indeed the case, we substitute $M_{r}$ and $M_{d}$ into the rate equation (2) and assume that no other processes contribute. The steady-state solution is

$$
\left[H_{2}\right]_{\mathrm{SS}}=\left(\frac{n_{H}}{2}\right)^{2}(\alpha+\beta)^{-1} .
$$


Figure 9 shows the steady-state fractional population of $\mathrm{H}_{2}$ versus $n_{H}$ for several values of $T_{R}$. As $n_{H}$ gets large, the ratio increases towards a limiting value of $1 / 2$ indicating that the gas has become completely molecular. This is not a realistic limit, however, because the time needed to reach the steady-state for quadrupole association is extremely long. The bottom panel of Figure 9 shows the time to reach one-half of the steady-state concentration

$$
t_{1 / 2}=\lambda^{-1} \ln \left(\frac{3 \alpha+\beta}{\alpha+\beta}\right) .
$$

This time scale is larger than the age of the universe for low temperatures and densities. At the higher temperatures needed to reach steady-state on a more realistic time scale, the fractional population of $\mathrm{H}_{2}$ drops substantially due to the increased efficiency of photodissociation. The bottom panel of Figure 9 shows that steady-state is obtainable within $10^{5}$ years for $T_{R} \geq 2500 \mathrm{~K}$ which corresponds to $n_{H} \approx 10^{3} \mathrm{~cm}^{-3}$. Using these numbers in the top panel shows that the fractional population of $\mathrm{H}_{2}$ is of order $10^{-12}$. For comparison, the formation mechanisms (49)-(52) yield a fractional population of $\mathrm{H}_{2}$ which is between $10^{-11}-10^{-15}$ when $500<z<1300$ [22]. The quadrupole mechanism cannot reach its steady-state limit for $T_{R}<2500 \mathrm{~K}$, so it is necessary to use the time-dependent solution (4) to estimate the population. The dashed curve in the top panel of Figure 9 shows this solution for $z=500-1300$ using the usual redshift formulas

$$
\begin{aligned}
& t=\left(14 \times 10^{9} \mathrm{yr}\right)(1+z)^{-3 / 2} \\
& T_{R}=(2.73 \mathrm{~K})(1+z) \\
& n_{H}=\left(10^{-6} \mathrm{~cm}^{-3}\right)(1+z)^{3} .
\end{aligned}
$$

All calculations used $M_{r}=10^{-28} \mathrm{~cm}^{3} / s$ so there is no $T_{M}$-dependence in the plots. Figure 8 shows this should be a good approximation for the values of $T_{R}$ considered. The fractional population is found to have a maximum near $z=800$ and a value of $10^{-13}$ at $z=1000$. The maximum represents the optimal balance between the density and time needed to recombine and the destructive efficiency of photodissociation. The results for this restricted model demonstrate that the quadrupole mechanism is competitive with the standard formation mechanisms (49)-(52) for these high redshifts and perhaps even dominates for $800<z<1300$.

\section{Conclusions}

A theoretical formalism is described for $\mathrm{H}_{2}$ which allows radiative association and photodissociation rate constants to be computed self-consistently for quadrupole transitions. The impact of extremely long-lived resonances is considered for LTE and non-LTE conditions that were prevalent in the early universe. Simple formulas are presented which may be used to compute the non-LTE concentration defects for any combination of matter and radiation temperature. It is found that the bound state defects $\delta_{b}$ at temperature $T_{M}$ yield a good approximation to a Boltzmann equilibrium distribution at temperature $T_{R}$ in the limiting case where inelastic collisions and charge transfer reactions are negligible. The unbound defects $\delta_{u}$ for the quasibound states were found to be quite large for $T_{M}<<T_{R}$ and $T_{M}>>T_{R}$, but do not substantially increase the formation rate constant due to the large exponential decay in the Boltzmann factor at small $T_{M}$ and the increasing value of $Q_{T}$ at large $T_{M}$. The photodissociation rate constant for quadrupole transitions is found to dominate the rate constants for other $\mathrm{H}_{2}$ photodestruction mechanisms for $T_{R} \leq 3000 \mathrm{~K}$. It is demonstrated that quadrupole association and dissociation of hydrogen is generally inefficient but may have occurred in the early universe for $z>500$. 
Quadrupole association and dissociation of hydrogen in the early universe

\section{Acknowledgments}

The author acknowledges support from NSF Grant No. PHY-1503615.

\section{References}

[1] Saslaw W C, and Zipoy D 1967 Nature 216976

[2] Peebles P J E, and Dicke R H 1968 Astrophysical Journal 154891

[3] Palla F, Salpeter E E, and Stahler S W 1983 Astrophysical Journal 271632

[4] Lepp S, Stancil P C, and Dalgarno A 2002 J. Phys. B: At. Mol. Phys. 35 R57-R80

[5] Kreckel H, Bruhns H, Cizek M, Glover S C O, Miller K A, Urbain X, and Savin D W 2010 Science $\mathbf{3 2 9} 69$

[6] Turner J, Kirby-Docken K, and Dalgarno A 1977 Astrophys. J. Supp. 35281

[7] Wolniewicz L, Simbotin I, and Dalgarno A 1998 Astrophys. J. Supp. 115293

[8] Forrey R C 2015 J. Chem. Phys. 143024101 URL http://dx.doi.org/10.1053/1.4926325/

[9] Forrey R C, Babb J F, Stancil P C, and McLaughlin B M submitted J. Phys. B

[10] Schwenke D W 1988 Theor. Chim. Acta 381

[11] Bromm V, and Loeb A 2003 Astrophysical Journal 59634

[12] Sugimura K, Omukai K, and Inoue A K 2014 Mon. Not. Roy. Astr. Soc. 445554

[13] Sugimura K, Coppola C M,Omukai K, Galli D, and Palla F 2016 Mon. Not. Roy. Astr. Soc. 456270

[14] Hummer D G, Rybicki G 1971 Annual Reviews 237

[15] De Jong T, Chu S-I, and Dalgarno A 1975 Astrophysical Journal 19969

[16] Bain R A and Bardsley J N 1972 J. Phys. B: At. Mol. Phys. 5 277 URL http://iopscience.iop.org/0022-3700/5/2/024/

[17] Forrey R C 2013 Phys. Rev. A 88 052709 URLhttp://dx.doi.org/10.1103/PhysRevA.88.052709/

[18] Bennett O J, Dickinson A S, Leininger T and Gadéa X 2003 Mon. Not. Roy. Astr. Soc. 341 361 URL http=//dx.doi.org/10.1046/j.1365-8711.2003.06422.x/

[19] Gutafsson M, Antipov S V, Franz J and Nyman G 2012 J. Chem. Phys. 137104301 URL http=//dx.doi.org/10.1063/1.4750029/

[20] Antipov S V, Gustafsson M and Nyman G 2013 Mon. Not. Roy. Astr. Soc. 430946 URL http://dx.doi.org/10.1093/mnras/sts615/

[21] Stancil P C, and Dalgarno A 1997 Astrophysical Journal 49076

[22] Coppola C M, Longo S, Capitelli M, Palla F, Galli D 2011 Astrophysical Journal Supplement Series 1937 
Table 1. Einstein A-coefficients $\left(\mathrm{s}^{-1}\right)$ for radiative transitions between quasibound and bound states of $\mathrm{H}_{2}$. The rotational level $j$ of the quasibound state and vibrational level $v$ of the bound state are arranged in columns. The coefficients for transitions to rotational levels $j-2, j, j+2$ are shown for each $v$.

\begin{tabular}{|c|c|c|c|c|c|c|c|}
\hline$v$ & $j=4$ & $j=13$ & $j=15$ & $j=17$ & $j=19$ & $j=21$ & $j=23$ \\
\hline & $4.25(-13)$ & $1.15(-10)$ & $3.04(-10)$ & $6.35(-10)$ & $1.20(-9)$ & $2.06(-9)$ & $3.03(-9)$ \\
\hline \multirow[t]{2}{*}{0} & $1.12(-14)$ & $3.92(-13)$ & $1.94(-12)$ & $7.17(-12)$ & $2.45(-11)$ & $8.20(-11)$ & $2.83(-10)$ \\
\hline & $7.12(-13)$ & $4.74(-12)$ & $9.41(-12)$ & $1.69(-11)$ & $3.19(-11)$ & $6.63(-11)$ & $1.59(-10)$ \\
\hline \multirow{3}{*}{1} & $7.46(-12)$ & $1.37(-9)$ & $3.42(-9)$ & $6.60(-9)$ & $1.12(-8)$ & $1.61(-8)$ & $1.64(-8)$ \\
\hline & $2.44(-15)$ & $1.19(-11)$ & $4.79(-11)$ & $1.55(-10)$ & $4.80(-10)$ & $1.51(-9)$ & $5.13(-9)$ \\
\hline & $8.34(-12)$ & $6.52(-11)$ & $1.30(-10)$ & $2.35(-10)$ & $4.51(-10)$ & $9.60(-10)$ & $2.01(-9)$ \\
\hline \multirow{3}{*}{2} & $6.62(-11)$ & $8.17(-9)$ & $1.88(-8)$ & $3.26(-8)$ & $4.69(-8)$ & $4.85(-8)$ & $1.55(-8)$ \\
\hline & $7.42(-13)$ & $1.53(-10)$ & $5.37(-10)$ & $1.58(-9)$ & $4.62(-9)$ & $1.43(-8)$ & $4.33(-8)$ \\
\hline & $4.76(-11)$ & $4.43(-10)$ & $8.96(-10)$ & $1.64(-9)$ & $3.17(-9)$ & $5.61(-9)$ & $5.30(-9)$ \\
\hline \multirow{3}{*}{3} & $3.94(-10)$ & $3.18(-8)$ & $6.60(-8)$ & $9.77(-8)$ & $1.04(-7)$ & $4.07(-8)$ & $4.61(-8)$ \\
\hline & $1.82(-11)$ & $1.18(-9)$ & $3.77(-9)$ & $1.05(-8)$ & $3.01(-8)$ & $8.05(-8)$ & $1.42(-7)$ \\
\hline & $1.71(-10)$ & $1.98(-9)$ & $4.07(-9)$ & $7.31(-9)$ & $1.10(-8)$ & $7.87(-9)$ & $1.46(-10)$ \\
\hline \multirow{3}{*}{4} & $1.76(-9)$ & $8.90(-8)$ & $1.59(-7)$ & $1.76(-7)$ & $7.91(-8)$ & $3.41(-8)$ & $9.74(-7)$ \\
\hline & $1.72(-10)$ & $6.42(-9)$ & $1.97(-8)$ & $5.31(-8)$ & $1.26(-7)$ & $1.86(-7)$ & 8.13(-8) \\
\hline & $4.13(-10)$ & $6.39(-9)$ & $1.24(-8)$ & $1.59(-8)$ & $8.15(-9)$ & $2.33(-11)$ & $8.08(-10)$ \\
\hline \multirow{3}{*}{5} & $6.21(-9)$ & $1.83(-7)$ & $2.46(-7)$ & $1.22(-7)$ & $2.10(-8)$ & $8.44(-7)$ & $1.54(-6)$ \\
\hline & $1.00(-9)$ & $2.80(-8)$ & $8.09(-8)$ & $1.72(-7)$ & $2.14(-7)$ & $7.43(-8)$ & $5.43(-11)$ \\
\hline & $6.44(-10)$ & $1.31(-8)$ & $1.60(-8)$ & $4.90(-9)$ & $1.06(-9)$ & $1.43(-9)$ & \\
\hline \multirow{3}{*}{6} & $1.80(-8)$ & $2.49(-7)$ & $1.50(-7)$ & $1.15(-8)$ & $6.72(-7)$ & $1.08(-6)$ & $1.34(-7)$ \\
\hline & $4.31(-9)$ & $9.45(-8)$ & $2.03(-7)$ & $2.14(-7)$ & $5.88(-8)$ & $6.74(-13)$ & \\
\hline & $4.62(-10)$ & $7.63(-9)$ & $5.15(-10)$ & 4.78(-9) & $2.21(-9)$ & & \\
\hline \multirow{3}{*}{7} & $4.46(-8)$ & $1.28(-7)$ & $6.53(-9)$ & $4.89(-7)$ & $6.85(-7)$ & $6.71(-8)$ & \\
\hline & $1.53(-8)$ & $1.78(-7)$ & $1.80(-7)$ & $3.87(-8)$ & $6.69(-11)$ & & \\
\hline & $6.13(11)$ & $2.07(-9)$ & $1.17(-8)$ & $2.88(-9)$ & & & \\
\hline \multirow{3}{*}{8} & $9.01(-8)$ & $4.65(-9)$ & $3.17(-7)$ & $3.87(-7)$ & $3.00(-8)$ & & \\
\hline & $4.41(-8)$ & $1.05(-7)$ & $1.93(-8)$ & $1.88(-10)$ & & & \\
\hline & $8.28(-9)$ & $1.65(-8)$ & $2.99(-9)$ & & & & \\
\hline \multirow{3}{*}{9} & $1.15(-7)$ & $1.56(-7)$ & $1.83(-7)$ & $1.14(-8)$ & & & \\
\hline & $7.86(-8)$ & $5.30(-9)$ & $2.64(-10)$ & & & & \\
\hline & $4.40(-8)$ & $1.97(-8)$ & & & & & \\
\hline \multirow{3}{*}{10} & $5.38(-8)$ & $5.88(-8)$ & $3.36(-9)$ & & & & \\
\hline & $5.42(-8)$ & $1.98(-10)$ & & & & & \\
\hline & $5.49(-8)$ & & & & & & \\
\hline \multirow{3}{*}{11} & $1.70(-9)$ & $5.78(-10)$ & & & & & \\
\hline & $6.54(-9)$ & & & & & & \\
\hline & $1.26(-8)$ & & & & & & \\
\hline \multirow{3}{*}{12} & $1.28(-9)$ & & & & & & \\
\hline & $2.61(-11)$ & & & & & & \\
\hline & $1.69(-10)$ & & & & & & \\
\hline \multirow{4}{*}{13} & $1.07(-10)$ & & & & & & \\
\hline & $9.82(-12)$ & & & & & & \\
\hline & $1.07(-14)$ & & & & & & \\
\hline & $6.71(-15)$ & & & & & & \\
\hline
\end{tabular}


Table 2. Same as Table 1 but for $j=24-29$.

\begin{tabular}{|c|c|c|c|c|c|c|c|}
\hline$v$ & $j=24$ & $j=25$ & $j=26$ & $j=27$ & $j=28$ & $j=29$ & $j=29$ \\
\hline & $4.46(-9)$ & $3.06(-9)$ & $1.79(-9)$ & $3.55(-10)$ & $8.65(-9)$ & $2.24(-7)$ & $1.95(-8)$ \\
\hline \multirow[t]{3}{*}{0} & $1.01(-9)$ & $1.08(-9)$ & $4.27(-9)$ & $4.44(-9)$ & $1.57(-8)$ & $3.14(-8)$ & $1.47(-8)$ \\
\hline & $4.36(-10)$ & $4.02(-10)$ & $8.13(-10)$ & $6.45(-10)$ & $2.92(-10)$ & $2.70(-11)$ & $1.46(-10)$ \\
\hline & $1.14(-8)$ & $3.74(-9)$ & $1.45(-8)$ & $4.06(-8)$ & $5.79(-7)$ & $2.95(-6)$ & $7.28(-7)$ \\
\hline \multirow[t]{3}{*}{1} & $1.76(-8)$ & $1.79(-8)$ & $5.14(-8)$ & $4.67(-8)$ & $5.95(-8)$ & $5.15(-9)$ & $4.32(-8)$ \\
\hline & $3.23(-9)$ & $2.47(-9)$ & $7.48(-10)$ & $3.37(-10)$ & $9.79(-11)$ & & \\
\hline & $1.25(-8)$ & $5.00(-8)$ & $7.52(-7)$ & $9.51(-7)$ & $3.05(-6)$ & $1.87(-6)$ & $2.89(-6)$ \\
\hline \multirow[t]{3}{*}{2} & $1.04(-7)$ & $9.17(-8)$ & $9.21(-8)$ & $6.44(-8)$ & $2.46(-9)$ & & \\
\hline & $9.83(-10)$ & $3.67(-10)$ & $3.05(-10)$ & & & & \\
\hline & $8.02(-7)$ & $1.02(-6)$ & $2.75(-6)$ & $2.54(-6)$ & $9.87(-7)$ & $2.58(-10)$ & $7.48(-7)$ \\
\hline \multirow[t]{3}{*}{3} & $1.16(-7)$ & $7.79(-8)$ & $1.92(-9)$ & $5.26(-10)$ & & & \\
\hline & $7.57(-10)$ & $3.97(-10)$ & & & & & \\
\hline & $2.27(-6)$ & $2.05(-6)$ & $6.01(-7)$ & $4.40(-7)$ & & & \\
\hline 4 & $1.24(-9)$ & $2.41(-10)$ & & & & & \\
\hline & $3.53(-7)$ & $2.49(-7)$ & & & & & \\
\hline \multicolumn{8}{|l|}{5} \\
\hline & $1.65(-11)$ & & & & & & \\
\hline 6 & & & & & & & \\
\hline
\end{tabular}

Table 3. Same as Table 1 but for $j=30-33$.

\begin{tabular}{llllllll}
\hline$v$ & $j=30$ & $j=31$ & $j=31$ & $j=32$ & $j=32$ & $j=33$ & $j=33$ \\
\hline & $2.93(-7)$ & $2.10(-6)$ & $3.69(-7)$ & $4.73(-6)$ & $2.15(-6)$ & $4.34(-6)$ & $2.14(-6)$ \\
0 & $2.52(-8)$ & $3.60(-9)$ & $1.90(-8)$ & & & & \\
& & & & & & & \\
\hline & $2.97(-6)$ & $2.87(-6)$ & $2.88(-6)$ & $3.60(-10)$ & $2.49(-6)$ & & \\
1 & $2.59(-9)$ & & & & & & \\
\hline & & $1.58(-6)$ & & & & & \\
2 & & & & & & \\
\hline
\end{tabular}

Table 4. Einstein A-coefficients $\left(\mathrm{s}^{-1}\right)$ for radiative transitions between quasibound states of $\mathrm{H}_{2}$. For rotational levels with more than one quasibound state, the entries are listed according to $j_{u_{f}}=j_{u_{i}}-2$ starting with the lowest $E_{u_{i}}$ and $E_{u_{f}}$, and ending with the $j_{u_{f}}=j_{u_{i}}$ transition.

\begin{tabular}{llllllllll}
\hline$j_{u_{i}}$ & $A_{u_{i} \rightarrow u_{f}}$ & $j_{u_{i}}$ & $A_{u_{i} \rightarrow u_{f}}$ & $j_{u_{i}}$ & $A_{u_{i} \rightarrow u_{f}}$ & $j_{u_{i}}$ & $A_{u_{i} \rightarrow u_{f}}$ & $j_{u_{i}}$ & $A_{u_{i} \rightarrow u_{f}}$ \\
\hline 15 & $1.29(-20)$ & 26 & $7.43(-11)$ & 30 & $5.08(-10)$ & 32 & $1.88(-11)$ & 33 & $1.47(-9)$ \\
17 & $4.50(-16)$ & 27 & $1.83(-10)$ & 31 & $4.96(-10)$ & 32 & $8.74(-10)$ & 33 & $8.56(-10)$ \\
19 & $8.10(-14)$ & 28 & $2.25(-10)$ & 31 & $5.17(-11)$ & 32 & $1.94(-9)$ & 34 & $3.88(-6)$ \\
21 & $1.60(-12)$ & 29 & $1.55(-10)$ & 31 & $1.24(-6)$ & 33 & $6.14(-10)$ & 34 & $1.01(-9)$ \\
23 & $1.24(-11)$ & 29 & $4.56(-10)$ & 31 & $9.19(-10)$ & 33 & $1.11(-11)$ & 35 & $3.34(-6)$ \\
25 & $5.66(-11)$ & 29 & $8.26(-10)$ & 31 & $1.01(-9)$ & 33 & $2.04(-6)$ & 35 & $1.64(-9)$ \\
\hline
\end{tabular}


Quadrupole association and dissociation of hydrogen in the early universe
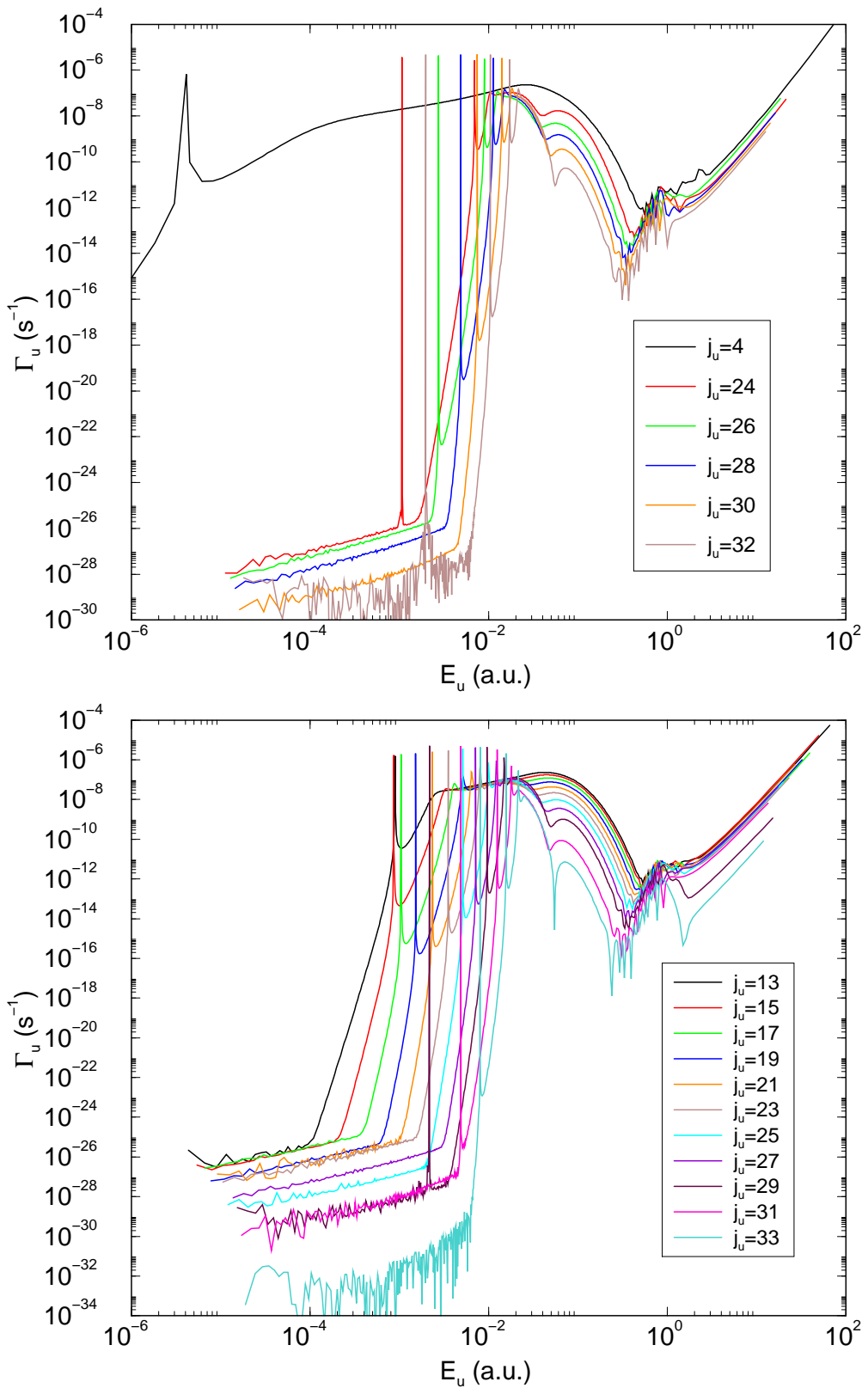

Figure 1. Radiative association width (42) for the formation of para- $\mathrm{H}_{2}$ (top) and ortho- $\mathrm{H}_{2}$ (bottom). Partial waves that support long-lived quasibound states are shown. The resonance peaks correspond to summed columns in Tables 1-3. 
Quadrupole association and dissociation of hydrogen in the early universe
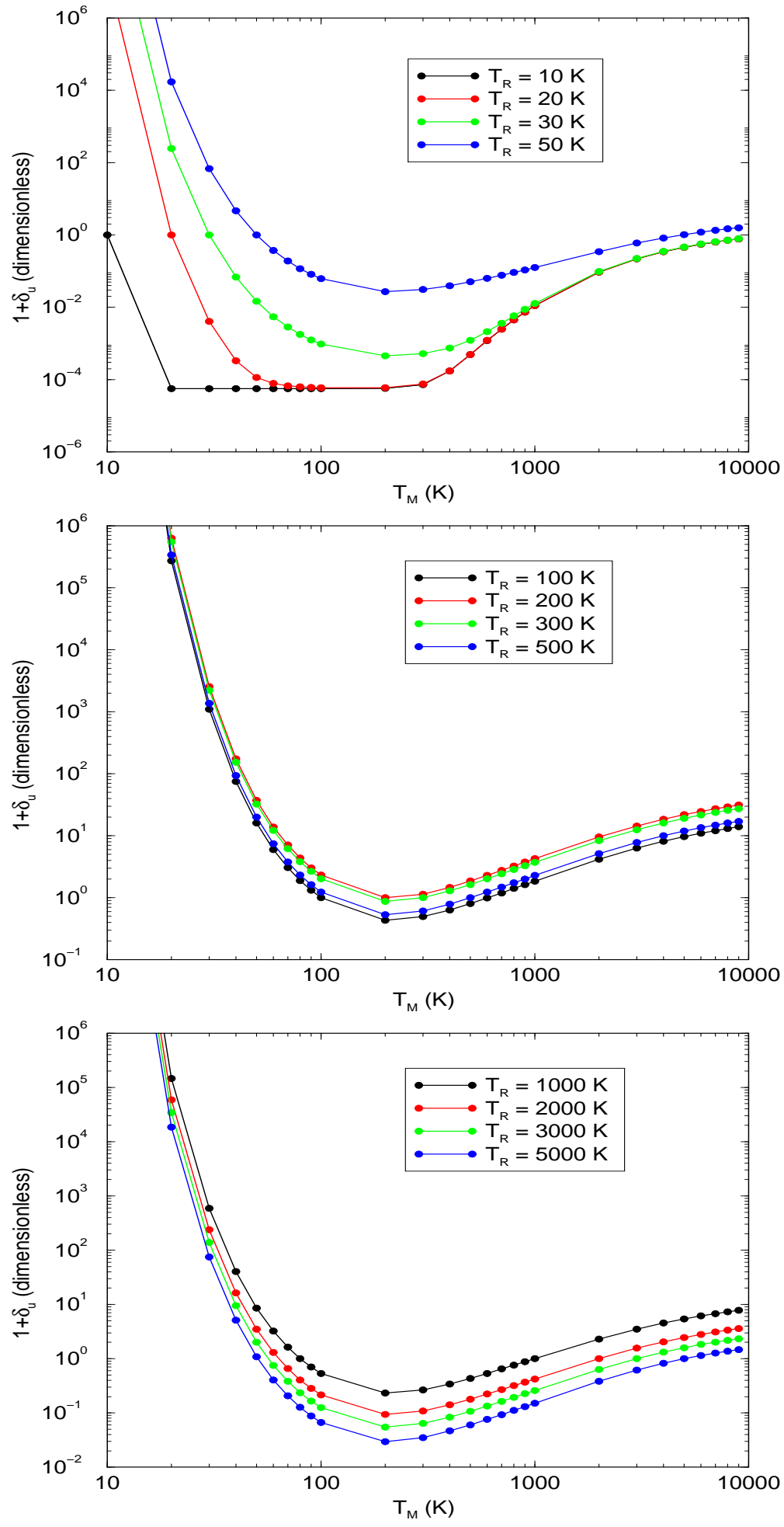

Figure 2. Non-LTE concentration factor for the $j=24$ quasibound state of $H_{2}$. Low values of $T_{R}$ tend to deplete the resonant state for $T_{M}>T_{R}$. Intermediate values of $T_{R}$ tend to increase the quasibound concentration beyond the LTE value, especially at low $T_{M}$. Large values of $T_{R}$ tend to deplete the quasibound state at intermediate $T_{M}$ while enhancing the concentration at low and high $T_{M}$. 
Quadrupole association and dissociation of hydrogen in the early universe
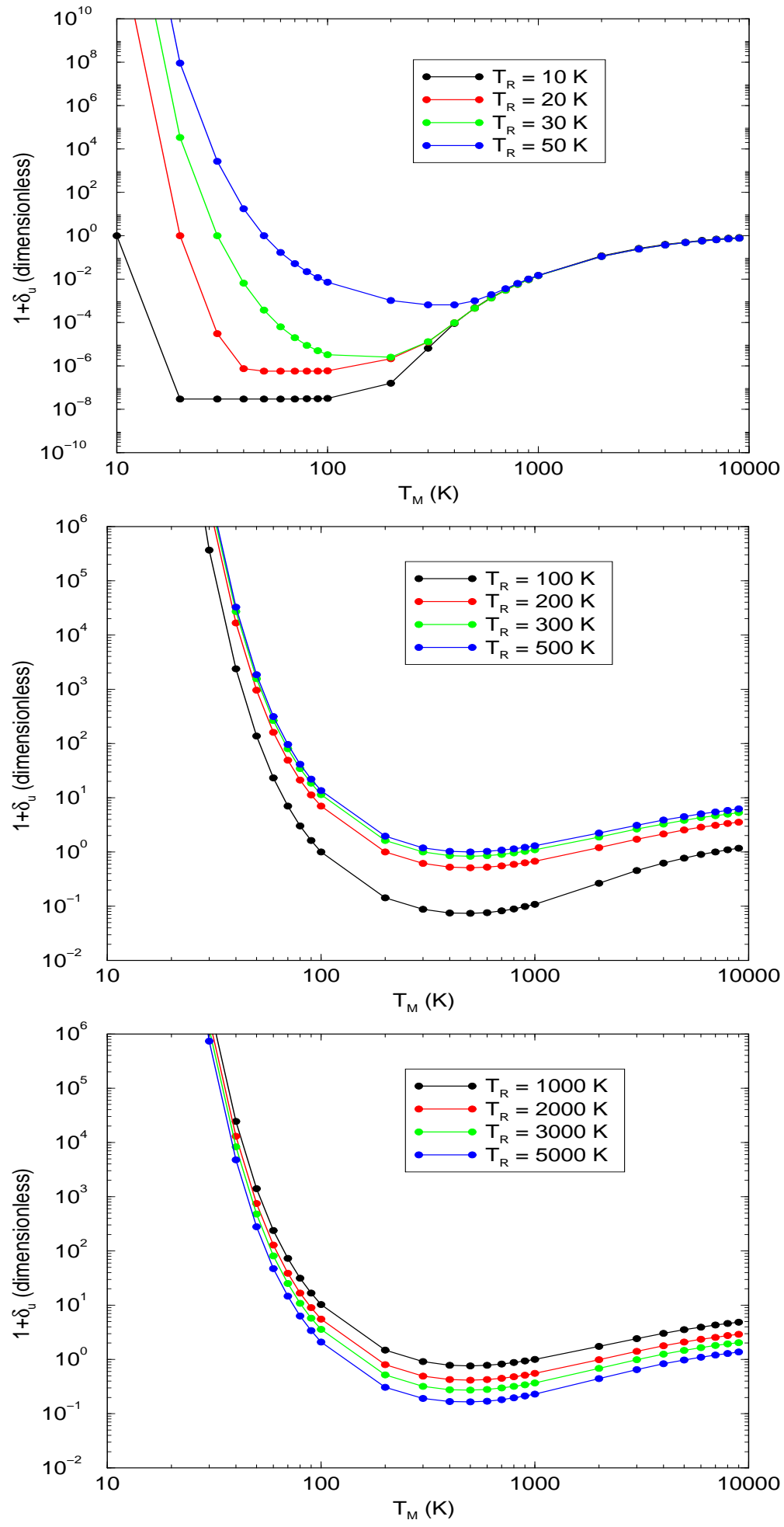

Figure 3. Same as Figure 2 except for $j=29$. 
Quadrupole association and dissociation of hydrogen in the early universe
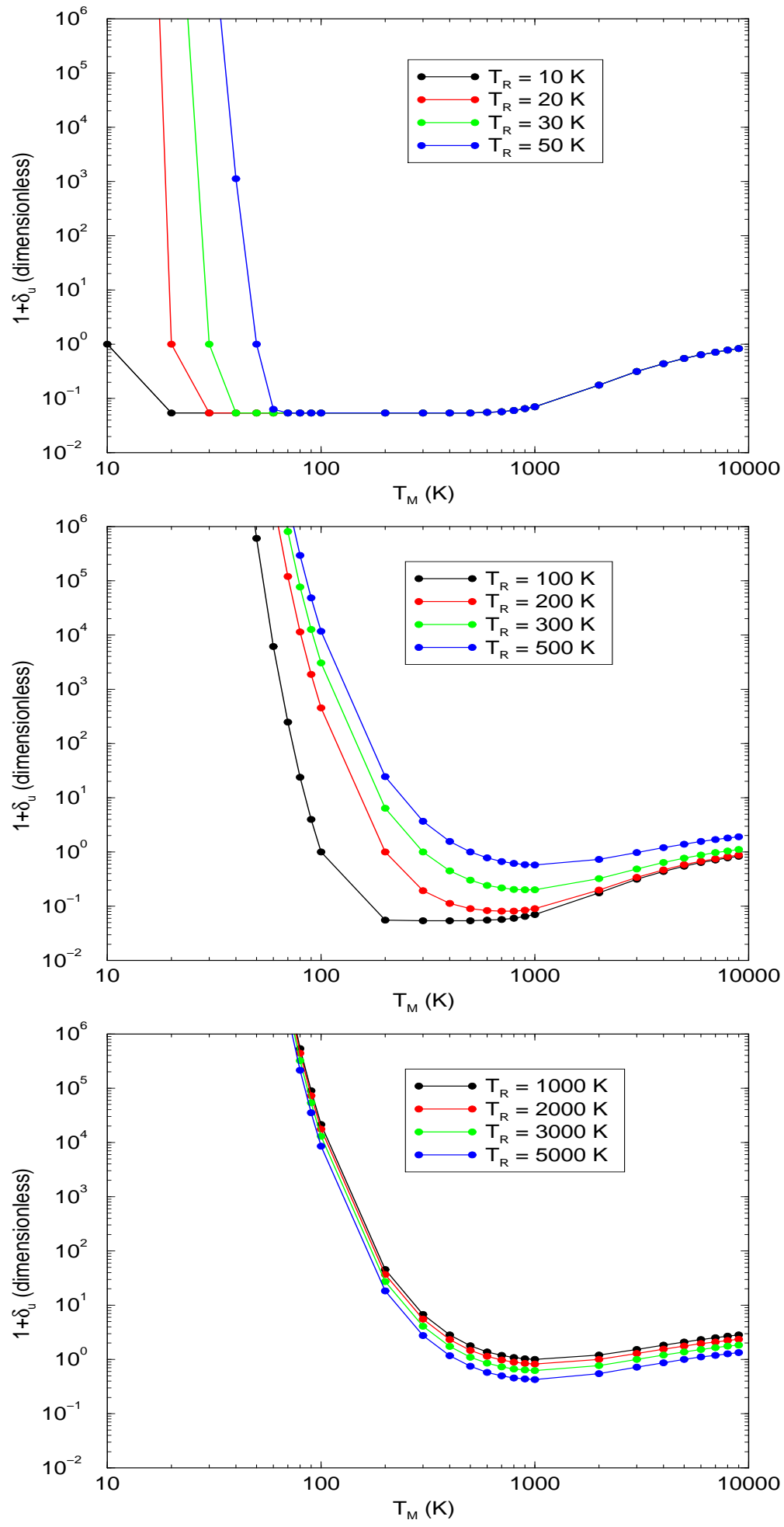

Figure 4. Same as Figure 2 except for $j=31$. 
Quadrupole association and dissociation of hydrogen in the early universe
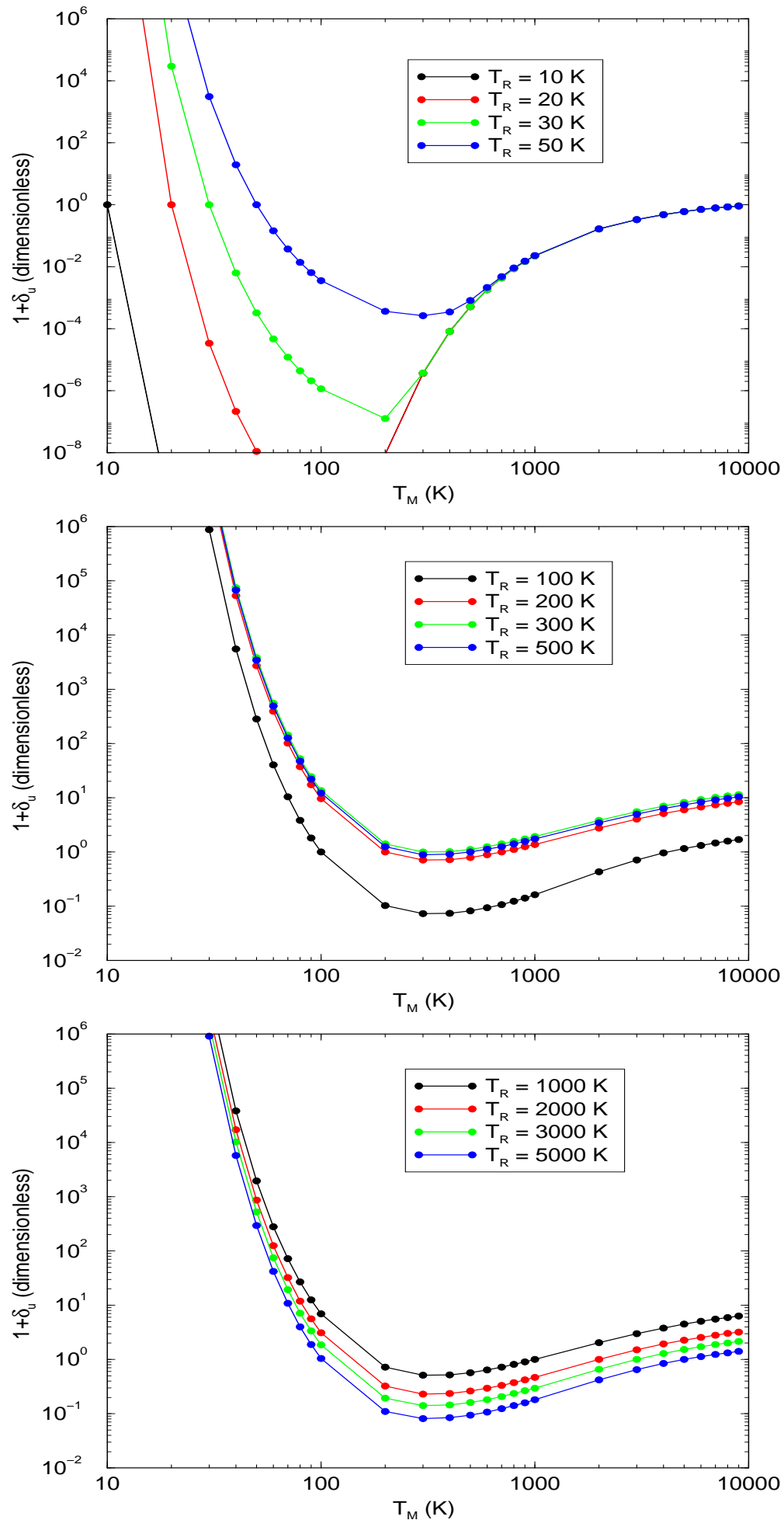

Figure 5. Same as Figure 2 except for $j=32$. 

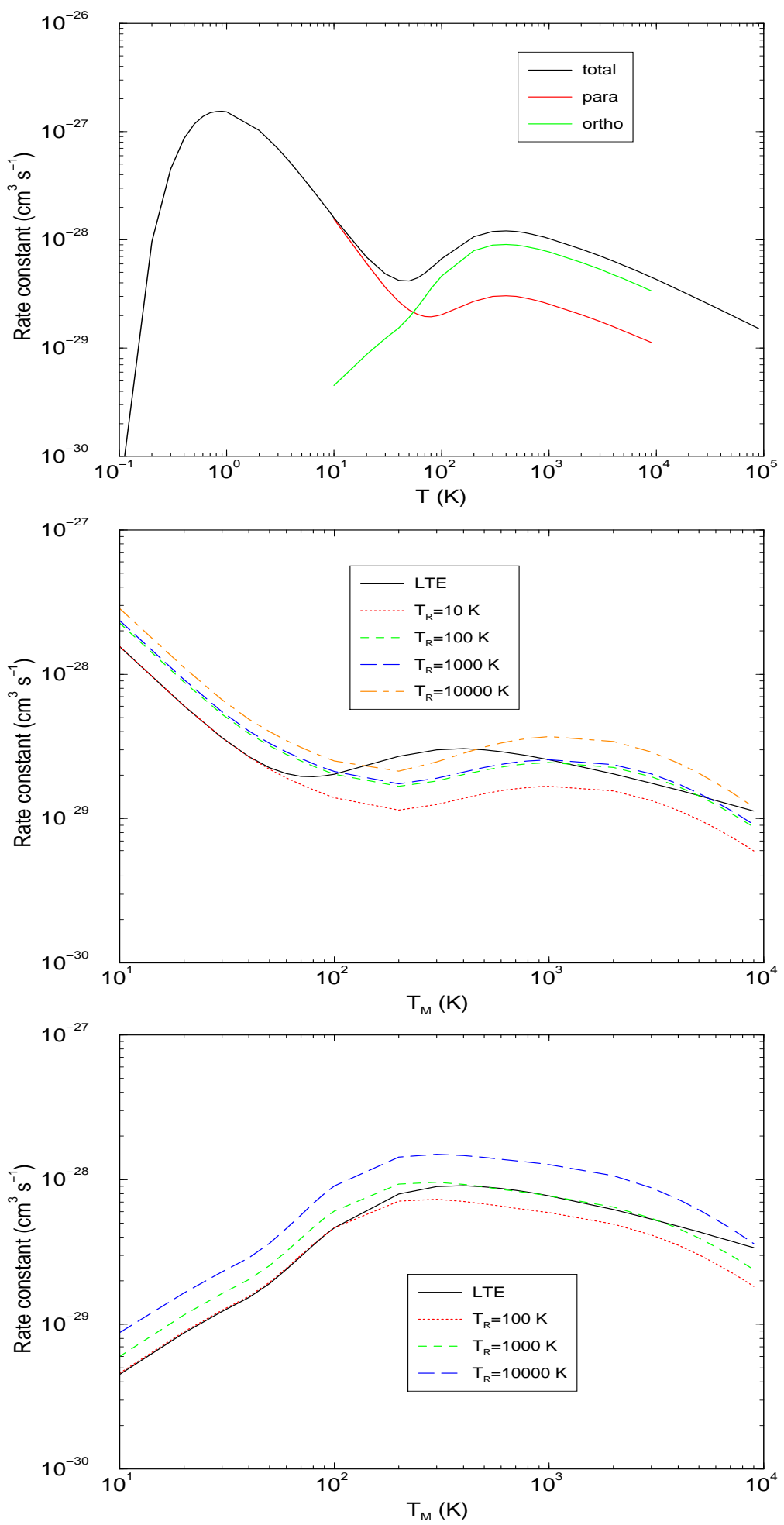

Figure 6. Rate constant for radiative association of $\mathrm{H}_{2}$ at LTE (top panel). Also shown are non-LTE rate constants as a function of $T_{R}$ and $T_{M}$ for forming para- $\mathrm{H}_{2}$ (middle panel) and ortho- $\mathrm{H}_{2}$ (bottom panel). 
Quadrupole association and dissociation of hydrogen in the early universe
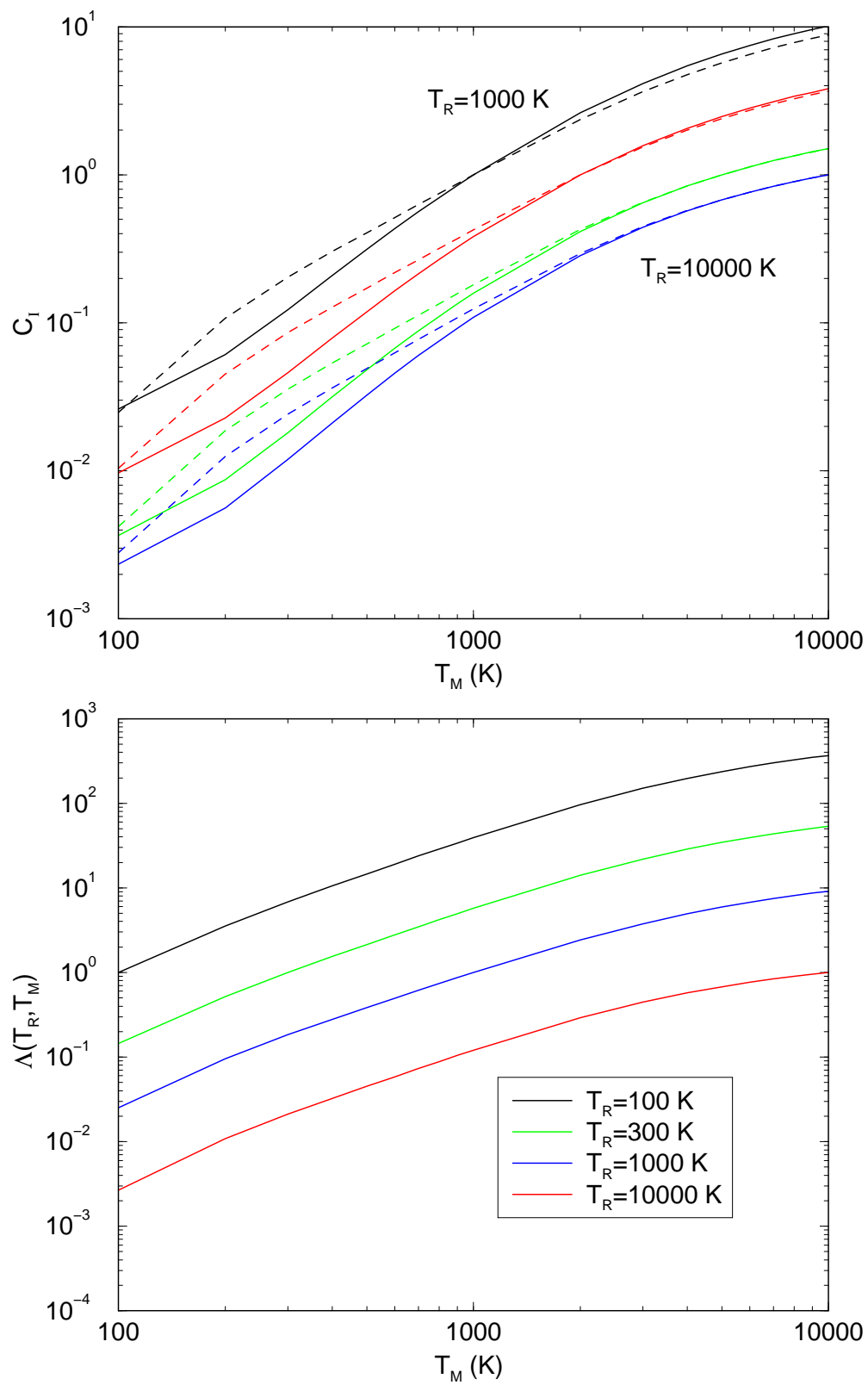

Figure 7. Symmetry constants $C_{I}$ (top panel) and scale factor $\Lambda$ (bottom panel). The symmetry constants are plotted for $T_{R}=1000,2000,5000$, and $10000 \mathrm{~K}$ using solid lines for $I=0$ and dashed lines for $I=1$. The scale factor is unity for $T_{R}=T_{M}$ but otherwise shows strong variation due to the assumed absence of inelastic three-body collisions. 

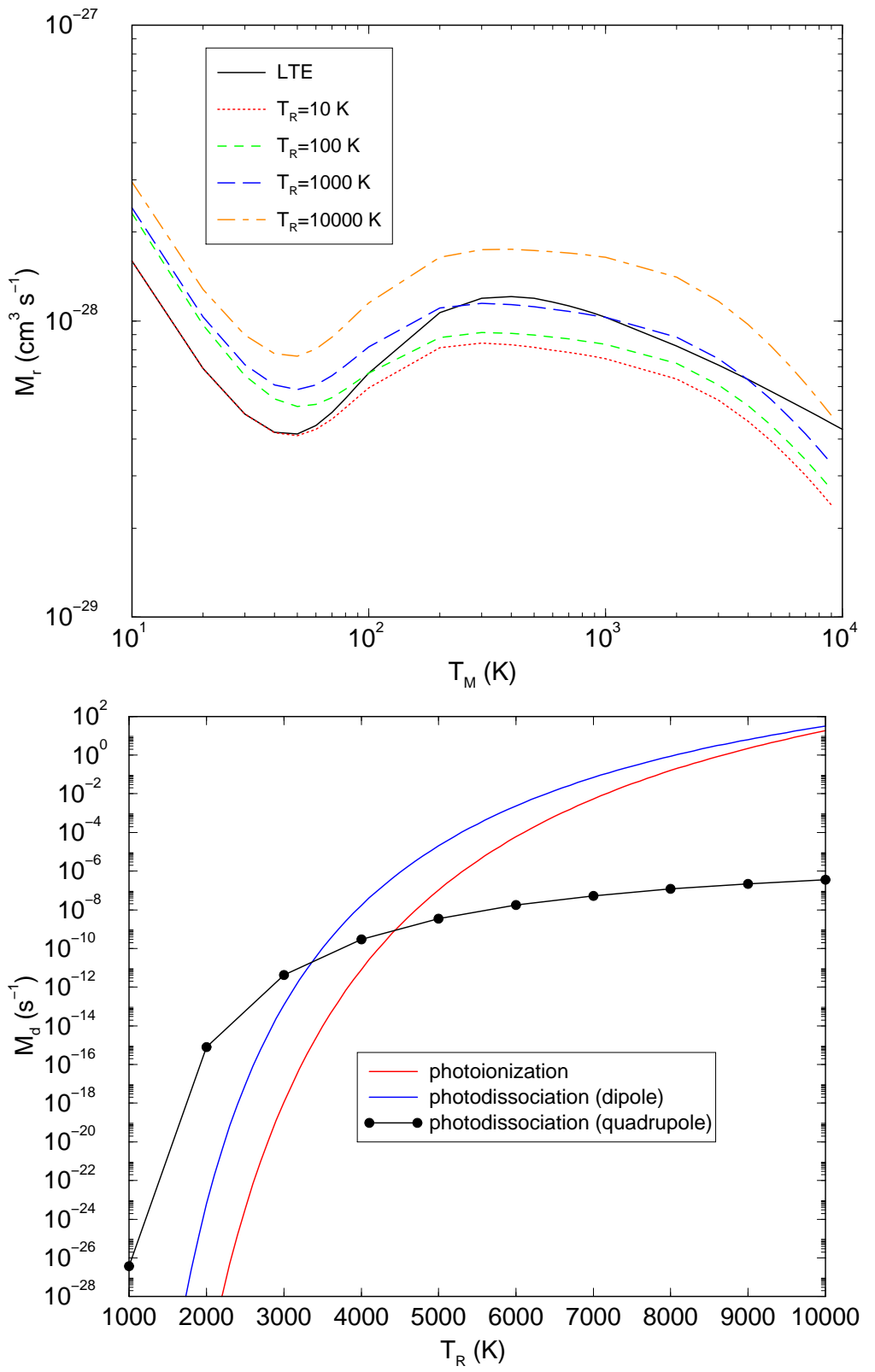

Figure 8. Steady-state rate constants $M_{r}$ (top panel) and $M_{d}$ (bottom panel). The dissociation rate constant has a very weak dependence on $T_{M}$ and is plotted as a function of $T_{R}$. Also shown on the bottom panel are rate constants for photoionization and indirect photodissociation (dipole) obtained from [22]. 

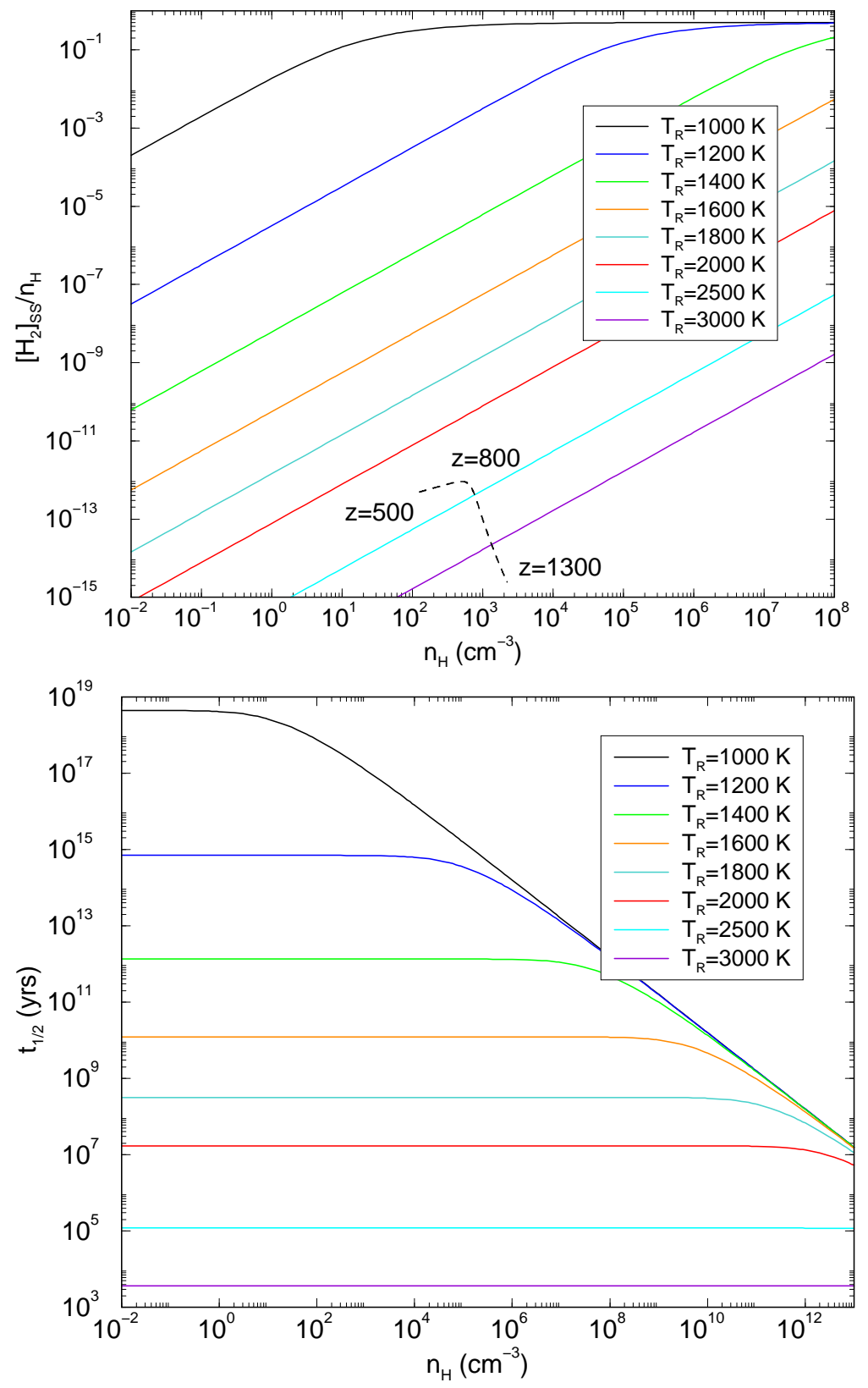

Figure 9. Steady-state fractional population of $\mathrm{H}_{2}$ (top panel) and the time required to reach one-half the steady-state value (bottom panel) for a system which considers only quadrupole association and dissociation of hydrogen. When $T_{R}<2500 \mathrm{~K}$, the system is not able to reach steady-state on a realistic time scale. In this case, the time-dependent solution (4) is shown as a dashed curve on the top panel for $500<z<1300$. 